PROCEEDINGS OF THE

AMERICAN MATHEMATICAL SOCIETY

Volume 139, Number 2, February 2011, Pages 559-569

S 0002-9939(2010)10493-8

Article electronically published on July 16, 2010

\title{
GEOMETRY OF QUASI-CIRCULAR DOMAINS AND APPLICATIONS TO TETRABLOCK
}

\author{
ŁUKASZ KOSIŃSKI \\ (Communicated by Franc Forstneric)
}

\begin{abstract}
We prove that the Shilov boundary is invariant under proper holomorphic mappings between some classes of domains (containing among others quasi-balanced domains with continuous Minkowski functionals). Moreover, we obtain an extension theorem for proper holomorphic mappings between quasi-circular domains.

Using these results we show that there are no non-trivial proper holomorphic self-mappings in the tetrablock. Another important result of our work is a description of Shilov boundaries of a large class of domains (containing among other the symmetrized polydisc and the tetrablock).

It is also shown that the tetrablock is not $\mathbb{C}$-convex.
\end{abstract}

\section{Introduction AND STATEMENT OF RESUlts}

In the paper we will use the notion of quasi-circular domains. Let $m_{1}, \ldots, m_{n}$ be relatively prime natural numbers. Recall that a domain $D \subset \mathbb{C}^{n}$ is said to be $\left(m_{1}, \ldots, m_{n}\right)$-circular (shortly quasi-circular) if

$$
\left(\lambda^{m_{1}} x_{1}, \ldots, \lambda^{m_{n}} x_{n}\right) \in D \quad \text { for any } \quad|\lambda|=1, x=\left(x_{1}, \ldots, x_{n}\right) \in D .
$$

If the relation (10) holds with $|\lambda| \leq 1$, then $D$ is said to be $\left(m_{1}, \ldots, m_{n}\right)$-balanced (shortly quasi-balanced).

Let $\mathcal{R}_{I I}$ denote the classical Cartan domain of the second type, i.e.

$$
\mathcal{R}_{I I}=\left\{z \in \mathcal{M}_{2 \times 2}(\mathbb{C}): z=z^{t},\|z\|<1\right\},
$$

where $\|\cdot\|$ is the operator norm and $\mathcal{M}_{2 \times 2}(\mathbb{C})$ denotes the space of $2 \times 2$ complex matrices. Put

$$
\Pi: \mathcal{M}_{2 \times 2}(\mathbb{C}) \ni z=\left(z_{i, j}\right) \rightarrow\left(z_{1,1}, z_{2,2}, \operatorname{det} z\right) \in \mathbb{C}^{3} .
$$

We define $\mathbb{E}:=\Pi\left(\mathcal{R}_{I I}\right)$. The domain $\mathbb{E}$ is called the tetrablock.

The tetrablock is a $(1,1,2)$-balanced domain in $\mathbb{C}^{3}$ that appears in control engineering and produces problems of a function-theoretic character. Its geometric properties have been investigated in several papers (see e.g. Ab-Wh-Yo,

Received by the editors November 11, 2009 and, in revised form, November 12, 2009 and March 10, 2010.

2010 Mathematics Subject Classification. Primary 32H35, 32A07.

Key words and phrases. Tetrablock, proper holomorphic mappings, group of automorphisms, quasi-circular domains, Shilov boundary.

This work was partially supported by the Research Grant of the Polish Ministry of Science and Higher Education No N N201 271435.

(C)2010 American Mathematical Society Reverts to public domain 28 years from publication 
Edi-Zwo2, You and references contained there). Recall here that in You the author obtained a description of the group of automorphisms of this domain using Kaup's theorem. In the paper we prove an Alexander-type theorem for the tetrablock showing that every proper holomorphic self-map of the tetrablock is an automorphism.

Theorem 1. Let $\varphi: \mathbb{E} \rightarrow \mathbb{E}$ be a proper holomorphic mapping. Then $\varphi$ is an automorphism.

As a side effect we obtain a natural correspondence between automorphisms of the tetrablock and of the classical domain of the second type indicated in Lemma 16 . This correspondence gives an easier and more elementary method of deriving the explicit formulas for automorphisms of the tetrablock. In particular, we extend results from $\mathrm{You}$ and simplify their proofs.

The methods used in the paper rely upon the investigation of proper holomorphic mappings between quasi-circular domains. We start with generalizing Bell's extension result (see [Bel2]). Next we analyze the behavior of the Shilov boundary under proper holomorphic mappings. We have the following:

Theorem 2. Let $D$ and $G$ be bounded domains in $\mathbb{C}^{n}$ and let $f: D \rightarrow G$ be a proper holomorphic mapping extending continously to $\bar{D}$. Assume that there is an increasing family of domains $\left\{G_{m}\right\}, G_{m} \subset \subset G_{m+1}$, such that $\bigcup G_{m}=G$ and $\overline{\left(\bigcup_{m} \partial_{s} G_{m}\right)} \cap \partial G=\partial_{s} G$. Then $f\left(\partial_{s} D\right)=\partial_{s} G$.

Note here that in general the Shilov boundary is not even invariant under biholomorphic polynomial mappings; see Example 10.

Based on the former idea we also obtain the following result:

Theorem 3. Let $f: D \rightarrow G$ be a proper holomorphic mappings between domains in $\mathbb{C}^{n}$. Let $L$ be a domain relatively compact in $G$. Put $K=f^{-1}(L)$. Then

$$
f\left(\partial_{s} K\right)=\partial_{s} L \quad \text { and } \quad f\left(\partial_{b} K\right)=\partial_{b} L .
$$

As a consequence of our considerations we will show that any proper holomorphic mapping between quasi-balanced bounded domains preserves the Shilov boundary. Namely, we have the following:

Corollary 4. a) Let $D \subset \mathbb{C}^{n}$ be a bounded domain and let $G$ be a bounded quasibalanced domain in $\mathbb{C}^{n}$. Assume that the Minkowski functional associated to $G$ is continuous and for any open, relatively compact subset $K$ of $D$ there is an open neighborhood $U$ of $\bar{D}$ such that $k_{D}(z, \bar{w})$ extends holomorphically to $U \times \tilde{K}$.

Then every proper holomorphic mapping $f: D \rightarrow G$ maps $\partial_{s} D$ onto $\partial_{s} G$.

b) Let $D$ and $G$ be bounded quasi-balanced domains. If the Minkowski functionals of $D$ and $G$ are continuous, then any proper holomorphic mapping between $D$ and $G$ preserves the Shilov boundary.

As we indicate in the sequel, the results obtained in the paper immediately give a description of the Shilov boundaries of many domains such as the symmetrized polydisc (see [Edi-Zwo1]), the tetrablock (see [Ab-Wh-Yo]), etc. Moreover, they exclude the existence of proper holomorphic mappings between some domains. For example, the well known theorem stating that there is no proper holomorphic mapping between the polydisc and the Euclidean ball is a direct consequence of our results. 
As a by-product of our considerations we obtain in Lemma 15] an extension of the main result from Tum-Hen].

In this paper in Remark 17 it is also shown that the tetrablock is not $\mathbb{C}$-convex. Recall that a consequence of the Lempert theorem is the fact that the Carathéodory pseudodistance and the Lempert function of a $\mathbb{C}$-convex domain with $\mathcal{C}^{2}$ boundary coincide (see $\mathrm{Jac}$ ). Since results obtained in [Ab-Wh-Yo (see also [Edi-Zwo2]) suggest that the equality between the Carathéodory pseudodistance and the Lempert function holds in the tetrablock, the tetrablock is the candidate for the first bounded pseudoconvex domain non-biholomorphically equivalent to a $\mathbb{C}$-convex domain for which the equality between the above-mentioned functions holds.

It also seems to be interesting whether the tetrablock may be exhausted by domains biholomorphic to $\mathbb{C}$-convex domains.

Here is some notation. Throughout the paper $\mathbb{D}$ denotes the unit disc in the complex plane. The unit Euclidean ball in $\mathbb{C}^{n}$ is denoted by $\mathbb{B}_{n}$. Moreover, $\operatorname{Prop}(D, G)$ is the set of proper holomorphic mappings between domains $D$ and $G$. The Shilov and Bergman boundaries are denoted respectively by $\partial_{s}$ and $\partial_{b}$.

I would like to thank Professor Włodzimierz Zwonek for reading the manuscript, his many remarks and fruitful discussions.

\section{EXTENSION OF PROPER HOLOMORPHIC MAPPINGS BETWEEN QUASI-BALANCED DOMAINS}

We start this section by recalling basic properties of circular domains and the Bergman projection, which will be useful in the sequel. By $k_{D}$ we shall denote the Bergman kernel associated to a domain $D$. Moreover, let $P_{D}$ denote the Bergman projection for $D$. We use the notation

$$
k_{D}^{\alpha}(z, w)=\partial^{\alpha} k_{D}(z, w) \quad \text { and } \quad k_{D}^{\bar{\alpha}}(z, w)=\partial^{\bar{\alpha}} k_{D}(z, w),
$$

where $\partial^{\alpha}$ stands for $\frac{\partial^{|\alpha|}}{z^{\alpha}}$ and $\partial^{\bar{\alpha}}$ stands for $\frac{\partial^{|\alpha|}}{\bar{w}^{\alpha}}$.

For a given $\left(m_{1}, \ldots, m_{n}\right)$-balanced domain $D$ in $\mathbb{C}^{n}$, where $m_{1}, \ldots, m_{n}$ are relatively prime natural numbers, we define the Minkowski functional

(3) $\mu_{D}(x):=\inf \left\{\lambda>0:\left(\lambda^{-m_{1}} x_{1}, \ldots, \lambda^{-m_{n}} x_{n}\right) \in D\right\}, \quad x=\left(x_{1}, \ldots, x_{m}\right) \in \mathbb{C}^{n}$.

This function has similar properties as the standard Minkowski functional for balanced domains. Some of them may be found in $\mathrm{Nik}$. In particular,

$$
\begin{aligned}
& \mu_{D}\left(\alpha^{m_{1}} x_{1}, \ldots, \alpha^{m_{n}} x_{n}\right)=|\alpha| \mu_{D}(x), \quad x \in \mathbb{C}^{n}, \alpha \in \mathbb{C}, \\
& D=\left\{x \in \mathbb{C}^{n}: \mu_{D}(x)<1\right\} .
\end{aligned}
$$

For a subset $K$ of $\mathbb{C}^{n}$ we put $\tilde{K}:=\{\bar{x}: x \in K\}$.

Remark 5 . Let $D$ be an $\left(m_{1}, \ldots, m_{n}\right)$-balanced bounded domain whose Minkowski functional is continuous. Put $D_{r}:=\left\{x \in \mathbb{C}^{n}: \mu_{D}(x)<r\right\}, r>0$. Since

$$
k_{D}\left(\left(r^{m_{1}} z_{1}, \ldots, r^{m_{n}} z_{n}\right), w\right)=k_{D}\left(z,\left(r^{m_{1}} w_{1}, \ldots, r^{m_{n}} w_{n}\right)\right)
$$

for $z, w \in D, r \in[0,1]$, we easily find that the function $(z, w) \rightarrow k_{D}(z, \bar{w})$ may be extended holomorphically to $D_{1 / r} \times \tilde{D}_{r}$ for any $0<r \leq 1$.

It follows from Bel1 that if $f: D \rightarrow G$ is a proper holomorphic mapping between bounded domains $D, G$ in $\mathbb{C}^{n}$, then for any $\Phi \in L^{2}(G)$ we have

$$
P_{D}\left(\operatorname{det}\left[f^{\prime}\right] \cdot(\Phi \circ f)\right)=\operatorname{det}\left[f^{\prime}\right] \cdot\left(\left(P_{G} \Phi\right) \circ f\right) .
$$


Assume additionally that $G$ is an $\left(m_{1}, \ldots, m_{n}\right)$-circular domain containing the origin. Choose $\delta>0$ such that $\delta \overline{\mathbb{B}_{n}} \subset G$. Let $\tilde{\theta}$ be a radial function in $\mathcal{C}_{0}^{\infty}\left(\delta \mathbb{B}_{n}\right)$ such that $\tilde{\theta} \geq 0$ and $\tilde{\theta}=1$ in the neighborhood of 0 . Since holomorphic functions assume their average values, we find that there is an $A>0$ such that for every $h \in \mathcal{O}(G) \cap L^{2}(G):$

$$
\partial^{\alpha} h(0)=\int_{G}\left(\partial^{\alpha} h\right) \theta d \lambda^{2 n}=\int_{G} h(-1)^{|\alpha|} \partial^{\alpha} \theta d \lambda^{2 n},
$$

where $\theta=A \tilde{\theta}$. On the other hand, $h(z)=\int_{G} k_{G}(z, w) h(w) d \lambda^{2 n}(w), z \in G$. Since $k_{G}(z, \cdot)$ extends holomorphically to a neighborhood of $\bar{G}$ provided that $z$ is sufficiently close to 0 , one may differentiate this formula at $z=0$ to get that

$$
\partial^{\alpha} h(0)=\int_{G} \partial^{\alpha} k_{G}(0, w) h(w) d \lambda^{2 n}(w), \quad h \in \mathcal{O}(G) \cap L^{2}(G) .
$$

This relation together with (7) gives

$$
P_{G}\left((-1)^{|\alpha|} \bar{\partial}^{\alpha} \theta\right)=k_{G}^{\bar{\alpha}}(\cdot, 0) .
$$

The next lemma has been proved by S. Bell in the case where $D$ and $G$ are bounded circular domains and $0 \in G$ (see $[\mathrm{Bel} 2]$ ). It is interesting that after minor modifications the methods used by Bell yield a stronger result. We present the whole proof for the sake of completeness.

Lemma 6. Let $D, G$ be bounded domains in $\mathbb{C}^{n}$. Suppose that $G$ is $\left(m_{1}, \ldots, m_{n}\right)$ circular and contains the origin. Assume moreover that the domain D satisfies the following property: for any open, relatively compact subset $K$ of $D$ there is an open set $U$ containing $\bar{D}$ such that $(z, w) \rightarrow k_{D}(z, \bar{w})$ extends holomorphically to $U \times \tilde{K}$.

Then any proper holomorphic mapping $f: D \rightarrow G$ extends holomorphically to a neighborhood of $\bar{D}$.

Proof. Let $m=\left(m_{1}, \ldots, m_{n}\right)$. Properties of the Bergman kernel and a standard argument imply that the equation

$$
k_{G}\left(\left(\lambda^{m_{1}} z_{1}, \ldots, \lambda^{m_{n}} z_{n}\right), w\right)=k_{G}\left(z,\left(\overline{\lambda^{m_{1}}} w_{1}, \ldots, \overline{\lambda^{m_{n}}} w_{n}\right)\right)
$$

holds for any $z, w \in G$ and $|\lambda|$ sufficiently close to 1 . Differentiating this formula several times with respect to $\bar{w}_{i}$ and putting $w=0$ we find that

$$
\frac{\partial^{\alpha} k_{G}}{\partial \bar{w}^{\alpha}}\left(\left(\lambda^{m_{1}} z_{1}, \ldots, \lambda^{m_{n}} z_{n}\right), 0\right)=\lambda^{\langle\alpha, m\rangle} \frac{\partial^{\alpha} k_{G}}{\partial \bar{w}^{\alpha}}(z, 0)
$$

for $\alpha \in \mathbb{N}^{n}, z \in G$ and $|\lambda|$ sufficiently close to 1 .

Whence a standard argument shows that there are $c_{\beta} \in \mathbb{C}$ such that

$$
k_{G}^{\bar{\alpha}}(z, 0)=\sum c_{\beta} z^{\beta}, z \in G,
$$

where the sum is taken over $\beta \in \mathbb{N}^{n}$ satisfying the relation $\langle\beta, m\rangle=\langle\alpha, m\rangle$. Therefore, the linear independence of $k_{G}^{\bar{\alpha}}(z, 0)$ (see (8) ) implies that for every $\beta \in \mathbb{N}^{n}$ there are $\tilde{c}_{\alpha}$ such that

$$
z^{\beta}=\sum \tilde{c}_{\alpha} k_{G}^{\bar{\alpha}}(z, 0),
$$

where the sum is taken over $\alpha \in \mathbb{N}^{n}$ satisfying the relation $\langle\alpha, m\rangle=\langle\beta, m\rangle$.

Now (13) together with (9) provides us with the function $\Phi_{i, k} \in \mathcal{C}_{0}^{\infty}\left(\delta \mathbb{B}_{n}\right)$ such that

$$
z_{i}^{k}=P_{G}\left(\Phi_{i, k}\right), \quad i=1, \ldots, n, k \in \mathbb{N} .
$$


Making use of the above relations we infer that

$\operatorname{det}\left[f^{\prime}(z)\right] f_{i}^{k}(z)=\operatorname{det}\left[f^{\prime}(z)\right]\left(z_{i}^{k} \circ f(z)\right)=\int_{D} k_{D}(z, w) \operatorname{det}\left[f^{\prime}(w)\right] \Phi_{i, k}(f(w)) d \lambda^{2 n}(w)$,

for $i=1, \ldots, n$, and $k \in \mathbb{N}$. From these relations and the assumption on $k_{D}$ we easily conclude that all the functions appearing in the left side of (15D) extend holomorphically to some open, connected neighborhood $U$ of $\bar{D}$.

We will briefly show that $f_{i}$ extends holomorphically to the domain $U$. Putting $u=\operatorname{det}\left[f^{\prime}\right]$ we have the following situation:

$$
u \in \mathcal{O}(U), \quad u \neq \equiv 0 \text { and } u f_{i}^{k} \in \mathcal{O}(U), k \in \mathbb{N} .
$$

Fix any point $x \in U$ such that $u(x)=0$. Changing the coordinate system, if necessary, we may assume that both $u$ and $u f_{i}$ satisfy the assumptions of the Weierstrass Preparation Theorem near $x$. Since $u f_{i}^{k}$ is holomorphic on $U$ for any $k \in \mathbb{N}$, one may easily check that the Weierstrass polynomial associated to $u$ divides the Weierstrass polynomial associated to $u f_{i}$. This, in particular, means that $f_{i}$ is locally bounded near the analytic set $\{u=0\}$, so the assertion follows from the Riemann Removable Singularity Theorem.

Remark 7. Note that the continuity of the Minkowski functional of a bounded quasibalanced domain $D$ is equivalent to the fact that for every $0<r<1$ the domain $D$ is relatively compact in $D_{1 / r}$. Therefore any quasi-balanced domain fulfills the assumptions of Lemma 6.

Corollary 8. Any proper holomorphic mapping $f: \mathcal{R}_{I I} \rightarrow \mathbb{E}$ may be extended holomorphically to a neighborhood of $\overline{\mathcal{R}_{I I}}$.

\section{Proofs of Theorems 2, 3 and applichtions}

We start this section with the following:

Remark 9. The technical assumption occurring in Theorem 2 seems to be very natural. Observe that $x \in \overline{\left(\bigcup_{m} \partial_{s} G_{m}\right)} \cap \partial G$ if and only if there is a subsequence $\left(n_{k}\right)$ and there are $x_{n_{k}} \in \partial_{s} G_{n_{k}}$ such that $x_{n_{k}} \rightarrow x$.

One may easily show that for any bounded domain $G$ and any increasing family of domains $\left\{G_{m}\right\}$ such that $\bigcup G_{m}=G$, the Shilov boundary of $G$ is contained in $\overline{\left(\bigcup_{m} \partial_{s} G_{m}\right)} \cap \partial G$.

Example 10. Note that Theorem 2 does not remain valid if we remove the assumption $\overline{\left(\bigcup_{m} \partial_{s} G_{m}\right)} \cap \partial G=\partial_{s} G$, even in the case where $f$ is a proper polynomial mapping. As an example one may take $D=\mathbb{D} \cap\{z \in \mathbb{D}: \operatorname{Im} z>0\}, G=\mathbb{D} \backslash[0,1)$ and $f(z)=z^{2}$.

Proof of Theorem 2. The inclusion $\partial_{s} G \subset f\left(\partial_{s} D\right)$ follows immediately from the definition of the Shilov boundary. We prove that $\partial_{s} D \subset f^{-1}\left(\partial_{s} G\right)$. Assume the contrary; i.e. there is a $\psi \in \mathcal{O}(D) \cap \mathcal{C}(\bar{D})$ such that

$$
\left|\psi\left(x_{0}\right)\right|>\max \left\{|\psi(x)|: x \in f^{-1}\left(\partial_{s} G\right)\right\},
$$

for some $x_{0} \in \partial D$. Note that

$$
\limsup _{m \rightarrow \infty} \max \left\{|\psi(x)|: x \in D \cap f^{-1}\left(\partial_{s} G_{m}\right)\right\} \leq \max \left\{|\psi(x)|: x \in \partial D \cap f^{-1}\left(\partial_{s} G\right)\right\} .
$$


Otherwise there would exist a subsequence $\left(m_{k}\right) \subset \mathbb{N}, \epsilon>0$ and $x_{m_{k}} \in D \cap$ $f^{-1}\left(\partial_{s} G_{m_{k}}\right)$ such that

$$
\left|\psi\left(x_{m_{k}}\right)\right|>\max \left\{|\psi(x)|: x \in \partial D \cap f^{-1}\left(\partial_{s} G\right)\right\}+\epsilon .
$$

Passing to a subsequence, if necessary, we can assume that $x_{m_{k}}$ converges to some $x_{0}$. Using the assumptions on the domain $G$ and the mapping $f$ we infer that $f\left(x_{0}\right) \in \partial_{s} G$. Thus

$$
\left|\psi\left(x_{0}\right)\right| \geq \max \left\{|\psi(x)|: x \in \partial D \cap f^{-1}\left(\partial_{s} G\right)\right\}+\epsilon \quad \text { and } \quad x_{0} \in \partial D \cap f^{-1}\left(\partial_{s} G\right),
$$

which gives an obvious contradiction.

Therefore we may take $m$ big enough and replace $x_{0}$ by a point $x_{0}^{\prime} \in f^{-1}\left(\bar{G}_{m}\right)$, sufficiently close to $x_{0}$, at which the mapping $f$ is non-degenerate so that

$$
\left|\psi\left(x_{0}^{\prime}\right)\right|>A:=\max \left\{|\psi(x)|: x \in D \cap f^{-1}\left(\partial_{s} G_{m}\right)\right\}, \quad \# f^{-1}\left(f\left(x_{0}^{\prime}\right)\right)=k,
$$

where $k$ denotes the multiplicity of $f$.

Let $h_{j}, j=1, \ldots, k$, be holomorphic mappings in the neighborhood of $f\left(x_{0}^{\prime}\right)$ given by $f^{-1}=\left\{h_{j}: j=1, \ldots, k\right\}$. Making use of (17) together with the Kronecker Theorem (see e.g. Har-Wri]) one may show the existence of a natural number $d$ such that

$$
\left|\psi\left(h_{1}\left(f\left(x_{0}^{\prime}\right)\right)\right)^{d}+\ldots+\psi\left(h_{k}\left(f\left(x_{0}^{\prime}\right)\right)\right)^{d}\right|>k A^{d} .
$$

To prove it, put $a_{j}=\psi\left(h_{j}\left(f\left(x_{0}^{\prime}\right)\right)\right), j=1, \ldots, k$. Change the order of $a_{j}$ if necessary so that $\left|a_{1}\right|=\ldots=\left|a_{l}\right|$ and $\left|a_{j}\right|<\left|a_{1}\right|$ for $j=l+1, \ldots, n$. Dividing all $a_{j}$ by $\psi\left(h_{1}\left(f\left(x_{0}^{\prime}\right)\right)\right)$ we find ourselves with the following situation:

$$
a_{j}=e^{i \theta_{j}}, j=1, \ldots, l, \quad\left|a_{j}\right|<1, j=l+1, \ldots, n \quad \text { and } \quad A<1,
$$

where $\theta_{j} \in \mathbb{R}, j=1, \ldots, l$.

Changing the order once again we may assume that $1, \theta_{1}, \ldots, \theta_{l_{1}}$ are $\mathbb{Q}$-linearly independent, $l_{1} \leq l, l_{1} \in \mathbb{N} \cup\{0\}$ and

$$
\theta_{j}=\frac{q_{j, 0}}{N}+\sum_{\iota=1}^{l_{1}} \frac{q_{j, \iota}}{N} \theta_{\iota},
$$

$j=l_{1}+1, \ldots, l$, where $q_{j, \iota} \in \mathbb{Z}, \iota=0, \ldots, l_{1}$, and $N \in \mathbb{N}$. Put $M=\max \left\{\left|q_{j, l}\right|, N\right\}$.

According to the Kronecker Theorem (see e.g. [Har-Wri]) there is a sequence of natural numbers $\left(\tilde{d}_{\mu}\right)$ such that $-\frac{1}{(\mu+1) k M}<\arg \left(e^{2 \pi i \tilde{d}_{\mu} \theta_{j}}\right)<\frac{1}{(\mu+1) k M}, j=1, \ldots, l_{1}$, $\mu \in \mathbb{N}$. In particular, $-\frac{1}{\mu+1}<\arg \left(e^{2 \pi i d_{\mu} \theta_{j}}\right)<\frac{1}{\mu+1}$ for $j=1, \ldots, l, \mu \in \mathbb{N}$, where $d_{\mu}:=N \tilde{d}_{\mu}$.

Properties of $\left(d_{\mu}\right)$ guarantee that $\left|a_{1}^{d_{\mu}}+\ldots+a_{l}^{d_{\mu}}\right| \rightarrow l$ as $\mu \rightarrow \infty$. Since $d_{\mu} \rightarrow \infty$, we find that $k A^{d_{\mu}} \rightarrow 0$ and $\left|a_{l+1}^{d_{\mu}}+\ldots+a_{k}^{d_{\mu}}\right| \rightarrow 0, \mu \rightarrow \infty$. Therefore $\mid a_{1}^{d_{\mu}}+\ldots+$ $a_{k}^{d_{\mu}} \mid-k A^{d_{\mu}} \rightarrow l>0$, which obviously proves the existence of a natural number $d$ fulfilling (18).

Put

$$
\zeta(x)=x_{1}^{d}+\ldots+x_{k}^{d}, \quad \text { for } \quad x=\left(x_{1}, \ldots, x_{k}\right) \in \mathbb{C}^{k} .
$$

A well known argument shows that the formula $\varphi=\zeta \circ(\psi \times \ldots \times \psi) \circ f^{-1}$ defines a holomorphic function on $G$. It follows from (17) and (18) that

$$
\left|\varphi\left(f\left(x_{0}^{\prime}\right)\right)\right|>\max \left\{|\varphi(x)|: x \in \partial_{s} G_{m}\right\},
$$

a contradiction. 
Remark 11. It is clear that the proof remains valid if the assumption $\overline{\left(\bigcup_{m} \partial_{s} G_{m}\right)} \cap$ $\partial G=\partial_{s} G$ occurring in Theorem 2 is replaced by a weaker condition $\overline{\left(\bigcup_{m} \partial_{b} G_{m}\right)} \cap$ $\partial G=\partial_{s} G$, where $\partial_{b}$ denotes the Bergman boundary.

The following result is a direct consequence of Theorem 2

Proposition 12. Let $D$ and $G$ be bounded domains in $\mathbb{C}^{n}$ and let $f: D \rightarrow G$ be a proper holomorphic mapping extending holomorphically to a neighborhood of $\bar{D}$. Assume that there is an increasing family of domains $\left\{G_{m}\right\}, G_{m} \subset \subset G_{m+1}$, such that $\bigcup G_{m}=G$ and $\overline{\left(\bigcup_{m} \partial_{b} G_{m}\right)} \cap \partial G=\partial_{b} G$. Then $f\left(\partial_{b} D\right)=\partial_{b} G$.

Proof. It follows from Remark 11 that $\partial_{s} G=\partial_{b} G$. Thus, Theorem 2 together with Remark 11 gives

$$
\partial_{b} D \subset \partial_{s} D \subset f^{-1}\left(\partial_{s} G\right)=f^{-1}\left(\partial_{b} G\right) .
$$

On the other hand, the inclusion $\partial_{b} G \subset f\left(\partial_{b} D\right)$ may be shown as in the proof of Theorem 2

Sketch of proof of Theorem 3, The inclusions $\partial_{s} L \subset f\left(\partial_{s} K\right)$ and $\partial_{b} L \subset f\left(\partial_{b} K\right)$ are clear.

It remains to show that $\partial_{s} K \subset f^{-1}\left(\partial_{s} L\right)$ and $\partial_{b} K \subset f^{-1}\left(\partial_{b} L\right)$. We will prove both inclusions simultaneously. Assume the contrary; i.e. there is a function $\psi \in$ $\mathcal{O}(K) \cap \mathcal{C}(\bar{G})($ resp. $\quad \psi \in \mathcal{O}(\bar{K}))$ such that $|\psi|$ does not attain its maximum on $f^{-1}\left(\partial_{b} L\right)$; i.e.

$$
\left|\psi\left(x_{0}\right)\right|>A:=\max \left\{|\psi(x)|: x \in f^{-1}\left(\partial_{s} L\right)\right\}
$$

(resp. $\left|\psi\left(x_{0}\right)\right|>A:=\max \left\{|\psi(x)|: x \in f^{-1}\left(\partial_{b} L\right)\right\}$ ) for some $x_{0} \in K$. Obviously $f\left(x_{0}\right)$ may be assumed to be a regular value of $f$.

Let $k$ denote the multiplicity of the mapping $f: D \rightarrow G$. Clearly, $\left.f\right|_{K}: K \rightarrow L$ is also of multiplicity $k$.

Write $f^{-1}=\left\{h_{1}, \ldots, h_{k}\right\}$ in a neighborhood of $a$, where $h_{i}$ are holomorphic functions. One may repeat the argument used in the proof of Theorem 2 to show the existence of a natural number $d$ such that

$$
\left|\psi\left(h_{1}\left(f\left(x_{0}\right)\right)\right)^{d}+\ldots+\psi\left(h_{k}\left(f\left(x_{0}\right)\right)\right)^{d}\right|>k A^{d} .
$$

Define $\zeta(x)=x_{1}^{d}+\ldots+x_{k}^{d}$, for $x=\left(x_{1}, \ldots, x_{k}\right) \in \mathbb{C}^{k}$. A function $\varphi$ given by the formula $\varphi=\zeta \circ(\psi \times \ldots \times \psi) \circ f^{-1}$ is holomorphic in $L$ and continuous on $\overline{f^{-1}(L)}=f^{-1}(\bar{L})$ (resp. $\varphi$ is holomorphic in an open neighborhood of $\bar{L}$ ). It follows from (20) that

$$
\left|\varphi\left(f\left(x_{0}\right)\right)\right|>\max \left\{|\varphi(x)|: x \in \partial_{s} L\right\},
$$

a contradiction.

Proof of Corollary 4, a) Define

$$
G_{m}:=\left\{x \in \mathbb{C}^{n}: \mu_{G}(x)<1-\frac{1}{m}\right\}, \quad m=2,3, \ldots
$$

It is clear that the family $\left\{G_{m}\right\}_{m}$ satisfies the assumptions of Theorem 2 So applying Lemma 6 we reduce the situation to the one occurring in Theorem 2

b) It is a direct consequence of $a$ ) and Remark 5] 
Remark 13. Note that Theorem 3 and Corollary 4 allow us to determine the Shilov boundary of some classes of domains containing the symmetrized polydisc (see Edi-Zwo1] $)$ and the tetrablock. For example, $\partial_{s} \mathbb{E}=\Pi\left(\partial_{s} \mathcal{R}_{I I}\right)=\Pi(\mathcal{U})$, where $\mathcal{U}$ consists of unitary symmetric matrices (see also [You, where the author, using elementary methods, computed the Shilov boundary of the tetrablock).

It is also interesting that Theorem 2 may be used for showing the non-existence of proper holomorphic mappings between some domains. For instance, using Corollary 4 we immediately see that $\operatorname{Prop}\left(\mathbb{D}^{n}, \mathbb{B}_{n}\right)$ and $\operatorname{Prop}\left(\mathbb{B}_{n}, \mathbb{D}^{n}\right)$ are empty for $n \geq 2$ (see also $[\mathrm{Nar}]$ ). As another example of the application of this result, observe that the theorem showing that there are no proper holomorphic mappings between $\mathbb{B}_{n} \times \mathbb{B}_{m}$ and $\mathbb{B}_{n+m}$ follows directly from Corollary 4

\section{Applichtions to the tetrablock}

The next result was proved in Rud2 for the Euclidean ball in $\mathbb{C}^{n}$. We would like to mention here that for our purposes a much weaker result of Tumanov and Henkin proved in Tum-Hen is sufficient. However, it seems interesting that after some modifications Rudin's idea may be applied to the symmetric domains.

First recall a well known classical result.

Lemma 14 (see Rud1, Theorem 8.1.2). Suppose that $\Omega_{1}$ and $\Omega_{2}$ are balanced domains in $\mathbb{C}^{n}$ and $\mathbb{C}^{m}$ respectively. Suppose moreover that $\Omega_{2}$ is convex and bounded and $F: \Omega_{1} \rightarrow \Omega_{2}$ is holomorphic. Then $F^{\prime}(0)$ maps $\Omega_{1}$ into $\Omega_{2}$.

If additionally $F(0)=0$, then $F\left(\lambda \Omega_{1}\right) \subset \lambda \Omega_{2}, 0 \leq \lambda \leq 1$.

Lemma 15. Let $a_{0}, b_{0}$ be any unitary symmetric matrices. Let $U, V$ be open neighborhoods of $a_{0}$ and $b_{0}$ respectively. Let $\varphi: U \cap \mathcal{R}_{I I} \rightarrow V \cap \mathcal{R}_{I I}$ be a biholomorphic mapping. If $\varphi\left(a_{k}\right) \rightarrow b_{0}$ for some $a_{k} \rightarrow a_{0}$, then $\varphi$ extends to an automorphism of $\mathcal{R}_{I I}$.

Proof. A direct computation shows that for any symmetric unitary matrix $a$ there is a unitary matrix $u$ such that $u u^{t}=a$. Since any of the mappings $\mathcal{R}_{I I} \ni x \rightarrow$ $u x u^{t} \in \mathcal{R}_{I I}$, where $u$ is unitary, is an automorphism of $\mathcal{R}_{I I}$, we may assume that $a_{0}=b_{0}=1$.

Recall that (see e.g. Hua]) for every $a \in \mathcal{R}_{I I}$ the mapping

$$
\varphi_{a}(x)=-a+\left(1-a a^{*}\right)^{1 / 2} x\left(1-a^{*} x\right)^{-1}\left(1-a^{*} a\right)^{1 / 2}
$$

is an automorphism of $\mathcal{R}_{I I}$, and $\varphi_{a}(0)=-a$ and its inverse is given by $\varphi_{a}^{-1}=\varphi_{-a}$.

Put $b_{k}=\varphi\left(a_{k}\right)$ and define $G_{k}:=\varphi_{b_{k}} \circ \varphi \circ \varphi_{-a_{k}}: \varphi_{a_{k}}\left(U \cap \mathcal{R}_{I I}\right) \rightarrow \varphi_{b_{k}}(V \cap$ $\left.\mathcal{R}_{I I}\right), \quad k \in \mathbb{N}$. Note that $G_{k}$ is a biholomorphic mapping, $G_{k}(0)=0$. Clearly $\varphi_{-a}(x) \rightarrow 1$ locally uniformly whenever $a \rightarrow 1$, so a compactness argument gives the existence of $\delta_{k}>0$ such that $\delta_{k} \rightarrow 1$ as $k \rightarrow \infty$, and both $\varphi_{a_{k}}\left(U \cap \mathcal{R}_{I I}\right)$ and $\varphi_{b_{k}}\left(V \cap \mathcal{R}_{I I}\right)$ contain a domain $\delta_{k} \mathcal{R}_{I I}$. Properly scaled, Lemma 14 implies that $\delta_{k}^{3} \leq\left|\operatorname{det} G_{k}^{\prime}(0)\right| \leq \delta_{k}^{-3}$.

Since $G_{k}(0)=0$, it follows that there exists a subsequence of $\left\{G_{k}\right\}$ (also denoted by $\left.\left\{G_{k}\right\}\right)$ converging locally uniformly to $G: \mathcal{R}_{I I} \rightarrow \mathcal{R}_{I I}$. Clearly $\left|\operatorname{det} G^{\prime}(0)\right|=1$ and $G(0)=0$, so by Lemma 14 the domain $\mathcal{R}_{I I}$ is mapped by $G^{\prime}(0)$ into $\mathcal{R}_{I I}$. Since $\left|\operatorname{det} G^{\prime}(0)\right|=1$, the mapping $G^{\prime}(0)$ preserves the volume. Hence $G^{\prime}(0)$ maps $\mathcal{R}_{I I}$ onto $\mathcal{R}_{I I}$; in particular, it is a unitary operator. Compose $G$ with $\left(G^{\prime}(0)\right)^{-1}$ and then apply the Cartan theorem in order to find that $G$ is also unitary. 
Let $\mathcal{N}=\left\{z \in \mathcal{M}_{2 \times 2}(\mathbb{C}): z=z^{t},\|z\| \neq \rho(z)\right\}$, where $\rho$ denotes the spectral radius. Note that $\mathcal{N} \cap \mathcal{R}_{I I}$ is open and dense in $\mathcal{R}_{I I}$. Moreover $\lambda z \in \mathcal{N}$ for any $z \in \mathcal{N}$ and $\lambda \in \mathbb{C} \backslash\{0\}$. For $z \in \mathcal{R}_{I I}$ define $D_{z}=\{\lambda z:\|\lambda z\|<1\} \subset \mathcal{R}_{I I}$.

Let $K$ be any compact subset of $\mathcal{N}$. Observe that

$$
\bigcup\left\{D_{z}: z \in K\right\} \subset \varphi_{a}\left(\mathcal{R}_{I I} \cap U\right)
$$

for $a \in \mathcal{R}_{I I}$ sufficiently close to 1 . Indeed, otherwise there would exist sequences $\left(\lambda_{n}\right) \subset \mathbb{C},\left(z_{n}\right) \subset K$ and $\left(a_{n}\right) \subset \mathcal{R}_{I I}$ such that $a_{n} \rightarrow 1$ and $\lambda_{n} \rightarrow \lambda_{0} \in \mathbb{C}, z_{n} \rightarrow$ $z_{0} \in K$ and $\lambda_{n} z_{n} \notin \varphi_{a_{n}}\left(\mathcal{R}_{I I} \cap U\right)$ (pass to subsequences, if necessary). If $\lambda_{0}=0$, then the contradiction is obvious. In the other case $\lambda_{0} z_{0} \in \mathcal{N} \cap \overline{\mathcal{R}_{I I}}$. It follows that $\operatorname{det}\left(1-\lambda_{0} z_{0}\right) \neq 0$ (otherwise $\rho\left(\lambda_{0} z_{0}\right) \geq 1 \geq\left\|\lambda_{0} z_{0}\right\|$ ). This in particular means that $\varphi_{a_{n}}^{-1}\left(\lambda_{n} z_{n}\right)$ converges to 1 (use formula (21)). Whence $\lambda_{n} z_{n} \in \varphi_{a_{n}}\left(\mathcal{R}_{I I} \cap U\right)$ for large $n$, a contradiction.

Since $G$ is unitary, $G^{-1}(\mathcal{N}) \cap \mathcal{N}$ is open and dense in $\mathcal{R}_{I I}$. Let $B=\{z \in$ $\left.\mathcal{M}_{2 \times 2}(\mathbb{C}): z=z^{t},\|z-p\|<2 c\right\}$, where $p=p^{t}$ and $c>0$ are chosen such that

$$
B \subset \subset G^{-1}(\mathcal{N}) \cap \mathcal{N} \text { and }\|z\|<1-c \text { for } z \in B .
$$

Property (22) yields the existence of an $n$ such that

$$
D_{z} \subset \varphi_{a_{n}}\left(U \cap \mathcal{R}_{I I}\right) \quad \text { and } \quad D_{G(z)} \subset \varphi_{b_{n}}\left(V \cap \mathcal{R}_{I I}\right), \quad z \in B .
$$

We may assume that for such an $n,\left\|G_{n}(z)-G(z)\right\|<c$ whenever $\|z\| \leq 1-c$, $z=z^{t}$.

Then for $z=z^{t}$ such that $\|z-p\|<c$, the set $D_{z}$ is contained in $\varphi_{a_{n}}\left(U \cap \mathcal{R}_{I I}\right)$. Since $\left\|G^{-1}\left(G_{n}(z)\right)-p\right\|=\left\|G_{n}(z)-G(p)\right\|<2 c$, we see that $G^{-1}\left(G_{n}(z)\right) \in B$. Therefore, making use of (23) we get that $D_{G_{n}(z)} \subset \varphi_{b_{n}}\left(V \cap \mathcal{R}_{I I}\right)$.

Thus we may apply a standard argument to the mapping $G_{n}: D_{z} \rightarrow \mathcal{R}_{I I}$, where $z=z^{t}$ is such that $\|z-p\|<c$, in order to find that $\left\|G_{n}(z)\right\| \leq\|z\|$. The same argument applied to $G_{n}^{-1}: D_{G_{n}(z)} \rightarrow \mathcal{R}_{I I}$ together with the previous inequality gives $\left\|G_{n}(z)\right\|=\|z\|$ for $\|z-p\|<c, z=z^{t}$. Obviously this equality remains valid on the whole of $\varphi_{a_{n}}\left(\mathcal{R}_{I I} \cap U\right)$.

Choose $r$ such that a ball $r \mathcal{R}_{I I}$ is contained in $\varphi_{a_{n}}\left(\mathcal{R}_{I I} \cap U\right) \cap \varphi_{b_{n}}\left(\mathcal{R}_{I I} \cap V\right)$ for a large $n$. The restriction of $G_{k}$ to $r \mathcal{R}_{I I}$ is an automorphism of $r \mathcal{R}_{I I}$ fixing 0 . Thus, from the description of the group of automorphism of the classical Cartan domain of the second type we conclude that $G_{k}$ is unitary. From this piece of information we immediately get the assertion.

We are ready to show the correspondence between proper holomorphic selfmappings of the tetrablock and the Cartan domain of the second type.

Lemma 16. Let $\varphi: \mathbb{E} \rightarrow \mathbb{E}$ be a proper holomorphic mapping. Then, there is a $\psi \in \operatorname{Aut}\left(\mathcal{R}_{I I}\right)$ such that

$$
\varphi \circ \Pi=\Pi \circ \psi .
$$

Proof of Lemma 16. First observe that $\Pi^{-1}(\mathbb{E})=\mathcal{R}_{I I}$, so it is very easy to see that $\Pi: \mathcal{R}_{I I} \rightarrow \mathbb{E}$ is proper. Put $f:=\varphi \circ \Pi$. By Corollary 8 the mapping $f$ extends to an open neighborhood $\Omega_{1}$ of $\overline{\mathcal{R}_{I I}}$. Define

$$
\mathcal{J}:=\left\{x \in \Omega_{1}: \operatorname{det}\left[f^{\prime}(x)\right] \neq 0 \text { and } f_{1}(x) f_{2}(x) \neq f_{3}(x)\right\} .
$$

Since every proper holomorphic mapping is non-degenerate, properties of the Shilov boundary show that the intersection of the sets $\mathcal{J}$ and $\partial_{s} \mathcal{R}_{I I}$ is non-empty. Take any $x_{0} \in \mathcal{J} \cap \partial_{s} \mathcal{R}_{I I}$. 
Fix a $y_{0}$ such that $\Pi\left(y_{0}\right)=f\left(x_{0}\right)$. The choice of $x_{0}$ and properties of covering maps allow us to choose open neighborhoods $U, V$ of $x_{0}, y_{0}$, respectively, and a biholomorphic mapping such that

$$
f=\Pi \circ \psi \quad \text { on } U \text {. }
$$

We find from Corollary 4 that $f\left(x_{0}\right)$ lies in the Shilov boundary of the tetrablock. So $\psi\left(x_{0}\right)$ is unitary.

Lemma 15 and the identity principle finish the proof.

Now we are able to prove an Alexander-type theorem for the tetrablock.

Proof of Theorem 1. It suffices to apply Lemma 16 to get that the mapping $\varphi \circ \Pi$ has multiplicity 2. Since $\Pi$ also has multiplicity 2 , we infer that $\varphi$ is an automorphism.

Remark 17. Note that the tetrablock is not $\mathbb{C}$-convex. Actually, let

$$
\gamma(x)=\left|x_{1}-\overline{x_{2}} x_{3}\right|+\left|x_{1} x_{2}-x_{3}\right|+\left|x_{3}\right|^{2}, \quad \text { for } \quad x=\left(x_{1}, x_{2}, x_{3}\right) \in \mathbb{C}^{3} .
$$

As shown in Ab-Wh-Yo, $x \in \mathbb{E}$ if and only if $\gamma(x)<1$.

For $\zeta \in \mathbb{C}$ put

$$
\varphi(\zeta):=\left(\frac{1-i}{2} \zeta+\frac{1+i}{2}, \frac{1+i}{2} \zeta+\frac{i-1}{2}, i \zeta\right) .
$$

Obviously $\varphi(1), \varphi(-1) \in \overline{\mathbb{E}}$. Moreover $\varphi(i \zeta)=\left(\frac{1+i}{2}(\zeta+1), \frac{i-1}{2}(\zeta+1),-\zeta\right)$. An easy computation shows that for any $\zeta \in \mathbb{R}$ :

$$
\begin{aligned}
\gamma(\varphi(i \zeta)) & =\left|\frac{1+i}{2}(\zeta+1)-\frac{i+1}{2}(\zeta+1) \zeta\right|+\left|-\frac{1}{2}(\zeta+1)^{2}+\zeta\right|+\zeta^{2} \\
& =\frac{\sqrt{2}}{2}\left|1-\zeta^{2}\right|+\left|\frac{\zeta^{2}+1}{2}\right|+\zeta^{2}=\frac{\sqrt{2}}{2}\left|1-\zeta^{2}\right|+\frac{3}{2} \zeta^{2}+1 / 2 .
\end{aligned}
$$

In particular $\gamma(\varphi(z))>1$ for any $z \in\{x \in \mathbb{C}: \operatorname{Re} x=0\}$, so $\mathbb{E} \cap \varphi(\mathbb{C})$ is not connected.

\section{REFERENCES}

[Ab-Wh-Yo] A. Abouhajar, M. White, and N. Young, A Schwarz lemma for a domain related to $\mu$-synthesis, Journal of Geometric Analysis 17 (2007), no. 4, 717-750. MR2365665 (2009e:32008)

[Bel1] S. Bell, Analytic hypoellipticity of the $\bar{\partial}-$ Neumann problem and extendability of holomorphic mappings, Acta Math. 147 (1981), no. 1-2, 109-116. MR631091(83a:32011)

[Bel2] S. Bell, Proper holomorphic mappings between circular domains, Comment. Math. Helv. 57 (1982), no. 4, 532-538. MR694605 (84m:32032)

[Edi-Zwo1] A. Edigarian and W. Zwonek, Geometry of the symmetrized polydisc, Arch. Math. 84 (2005), 364-374. MR2135687 (2006b:32020)

[Edi-Zwo2] A. Edigarian and W. Zwonek, Schwarz lemma for the tetrablock, Bulletin of the London Mathematical Society 41 (2009), no. 3, 506-514. MR2506834(2010e:32007)

[Har-Wri] G. H. Hardy and E. M. Wright, An Introduction to the Theory of Numbers, Oxford Science Publ., 1978. MR0067125 (16:673c)

[Hen-Nov] G. M. Henkin and R. Novikov, Proper mappings of classical domains, Lecture Notes in Math. 1043, Springer-Verlag, 1984, pp. 625-627.

[Hua] L. K. Hua, Harmonic Analysis of Functions of Several Complex Variables in the Classical Domains, Amer. Math. Soc., Providence, RI, 1963. MR0171936 (30:2162)

[Jac] D. Jacquet, $\mathbb{C}$-convex domains with $C^{2}$ boundary, Complex Var. Elliptic Equ. 51 (2006), no. 4, 303-312. MR2218722 (2007j:32005) 
[Lem] L. Lempert, La métrique de Kobayashi et la représentation des domaines sur la boule, Bull. Soc. Math. France 109 (1981), 427-474. MR660145 (84d:32036)

[Mok] N. Mok, Nonexistence of proper holomorphic maps between certain classical bounded symmetric domains, Chinese Annals of Mathematics - Series B 29 (2008), no. 2, 135-146. MR 2392329(2009i:32024)

[Nar] R. Narasimhan, Several Complex Variables, Chicago Lectures in Mathematics, 1971. MR0342725 (49:7470)

[Nik] N. Nikolov, The symmetrized polydisc cannot be exhausted by domains biholomorphic to convex domains, Ann. Polon. Math. 88 (2006), 279-283. MR2260407 (2007f:32024)

[Rud1] W. Rudin, Function theory in the unit ball of $\mathbb{C}^{n}$, Grundlehren der Mathematischen Wissenschaften, 241, Springer-Verlag, 1980. MR601594 (82i:32002)

[Rud2] W. Rudin, Holomorphic maps that extend to automorphisms of a ball, Proc. Amer. Math. Soc. 81 (1981), no. 3, 429-432. MR597656 (82c:32012)

[Tu1] Z.-H. Tu, Rigidity of proper holomorphic mappings between equidimensional bounded symmetric domains, Proc. Amer. Math. Soc. 130 (2002), 1035-1042. MR1873777 (2003a:32027)

[Tu2] Z.-H. Tu, Rigidity of proper holomorphic mappings between nonequidimensional bounded symmetric domains, Math. Z. 240 (2002), 13-35. MR1906705 (2003g:32034)

[Tu3] Z.-H. Tu, Rigidity of proper holomorphic mappings between bounded symmetric domains, Geometric function theory in several complex variables, pp. 310-316, World Sci. Publishing, River Edge, NJ, 2004. MR2115800 (2005m:32032)

[Tum-Hen] A. E. Tumanov and G. M. Khenkin, Local characterization of holomorphic automorphisms of Siegel domains, Funktsional. Anal. i Prilozhen. 170 (1983), no. 4, 49-61 (Russian). MR725415 (86a:32063)

[You] N. Young, The automorphism group of the tetrablock, Journal of the London Mathematical Society 77 (2008), no. 3, 757-770. MR2418303 (2009a:32032)

Instytut Matematyki, Uniwersytet Jagielloński, Łojasiewicza 6, 30-348 Kraków, POLAND

E-mail address: lukasz.kosinski@im.uj.edu.pl 